\title{
A NOTE ON $\Lambda$-BANACH FRAMES AND O-FRAMES
}

\author{
M. P. GOSWAMI ${ }^{\dagger}$ AND H. K. PATHAK
}

$\begin{array}{lll}\text { Date of Receiving } & : & 06.02 .2019 \\ \text { Date of Revision } & : & 29.08 .2019 \\ \text { Date of Acceptance } & : & 09.12 .2019\end{array}$

\begin{abstract}
In this paper, we define and study near-exact $\Lambda$-Banach frame for operator spaces with some illustrative examples. A sufficient condition for a $\Lambda$ Banach frame to be near-exact has been given. In the sequel, we deal with a Krien-Milman-Rutman type stability result for $\Lambda$-Banach frame. Moreover, we demonstrate a method to construct $\Lambda$-Banach frames associated with O-frames. Also, some results regarding characterizations of O-frames have been given.
\end{abstract}

\section{Introduction}

Frames for Hilbert spaces were introduced by Duffin and Schaeffer [5] In 1952. Recall that, a sequence $\left\{x_{n}\right\}_{n=1}^{\infty}$ of elements in a Hilbert space $H$ is said to be a frame for $H$ if there exist constants with $0<A_{1} \leq A_{2}<\infty$ such that

$$
A_{1}\|x\|^{2} \leq \sum_{n=1}^{\infty}\left|\left\langle x, x_{n}\right\rangle\right|^{2} \leq A_{2}\|x\|^{2}, \quad x \in H
$$

Nowadays, frames play an important role in many applications in mathematics and engineering. In particular, frames are widely used in wavelet theory, signal processing, wireless sensor network, image processing and many more. In view of theoretical developments, many concepts and notions from Banach spaces and operator theory are used to study frames.

In 1991, Hilbert frame was extended to Banach spaces by Grochenig [7] who introduced the notion of Banach frame in Banach spaces. Since then a number of generalizations and variations of frames in Banach spaces have been introduced and studied by various authors, namely, retro Banach frames [8], fusion Banach frames [10], operator frames [13], operator Banach frames [16] etc. Besides, reconstruction property in the context of frames in Banach spaces was studied in [1] and [11]. In 2015, O. Reinov

2010 Mathematics Subject Classification. 42C15, 42A38.

Key words and phrases. Banach frame, $\Lambda$-Banach frame, O-frame.

The authors are indebted to the anonymous referees for their helpful comments and suggestions toward the improvement of the paper.

Communicated by: L. K. Vashisht

${ }^{\dagger}$ Corresponding author. 